\title{
Observation of Ultrastructure in Nasal Mucosa on Allergic Perennial Rhinitis Cases before and after the Operations
}

\author{
Weinian Lin, Jun Liao, Jinchao Lin, Zhongzhu Nian, Xiaodong Zhang, Xiaoming Ye \\ Department of Otorhinolaryngology, Quanzhou First Affiliated Hospital of Fujian Medical University, Quanzhou, China \\ Email: linwn99@163.com
}

How to cite this paper: Lin, W.N., Liao, J., Lin, J.C., Nian, Z.Z., Zhang, X.D. and Ye, X.M. (2016) Observation of Ultrastructure in Nasal Mucosa on Allergic Perennial Rhinitis Cases before and after the Operations. International Journal of Otolaryngology and Head \& Neck Surgery, 5, 181-188. http://dx.doi.org/10.4236/ijohns.2016.55029

Received: March 24, 2016

Accepted: September 17, 2016

Published: September 22, 2016

Copyright $\odot 2016$ by authors and Scientific Research Publishing Inc. This work is licensed under the Creative Commons Attribution International License (CC BY 4.0).

http://creativecommons.org/licenses/by/4.0/

\begin{abstract}
Objective: The partial inferior turbinectomy and septoplasty was applied to treat the allergic perennial rhinitis (APR), and to observe the ultrastructure changes of the nasal mucosa before and after the operations. Methods: For 36 cases of research objects diagnosed with APR, the partial inferior turbinectomy and septoplasty was administered. For 6 APR cases among them, the pre- and postoperative observation of anterior nasal mucosa of the inferior turbinate on the same side under the electron microscope was conducted for one year respectively. In addition, their pathological alterations of tissues were also conducted. Results: In the pre-operative observation under the electron microscope, it was found that the nasal mucosae epithelium cells were nude without cilia. The lamina propria had edema, and mesenchyme presented the infiltration of substantial eosinophilic granulocytes, basophilic granulocytes, plasmacytes and mast cells. Additionally, abundant degranulation and vacuolation of cytoplasts were observed. The plentiful glands, duct ectasia, edema and structural changes were also found. Some gland cells had degenerated. After the operation, it was found that the epithelium nudity still existed and the deficiency of cilia was not improved. Only a very small amount of microvilli existed. The edema of lamina propria was improved and eosinophilic granulocytes were rarely observed in mesenchyme. However, the infiltration of basophilic granulocytes, plasmocytes and mast cells was still observed. The particle structure was generally stable and the central crystal was clear without degranulation. Meanwhile, the amount of glands was reduced and the tissue structure tended to be recovered. Overall, the nasal mucosa showed changes as chronic inflammation. Conclusions: For the treatment of APR with the methods presented by our research institution, in one year before and after the operation, ultrastructural changes of inferior turbinate mucosa tissues were observed from the preoperatively pathological changes of typical APR to the chronic inflammation with the primary infiltration of neutrophilic granulocyte and mast
\end{abstract}


cells.

\section{Keywords}

Rhinitis, Allergic, Perennial, Nasal Mucosa, Microscope, Electronic, Histopathology

\section{Introduction}

Allergic rhinitis (AR) is common Otorhinolaryngology department. Operation is not its major treatment method, but is adopted by some scholars in some patients with AR combined with structural abnormalities like deflection of nasal septum, and the methods vary [1]-[4]. There are also reports on treating allergic perennial rhinitis (APR) with partial inferior turbinectomy and septoplasty [1]-[3], of which short-term curative effect is satisfying. In this research, partial inferior turbinectomy and septoplasty were used to treat allergic perennial rhinitis (APR), and the observation of the ultrastructure of inferior turbinate mucosa before and after operation was also conducted, aiming to find whether there was any change of nasal mucosa ultrastructure as APR patients' clinical symptoms were improved after operation.

\section{Data and Method}

\subsection{Clinical Data}

According to the diagnosis criteria revised by Otorhinolaryngology branch of Chinese Medical Association in Lan Zhou, 2004 [4], typical disease history, symptoms, signs, together with significant allergen skin prick test (SPT) were adopted to collect 36 cases of APR patients with deflection of nasal septum from October, 2005 to April, 2008. There are 19 cases of male and 17 cases of female, aging from 18 to 54, with an average age of 26, medical history ranging from 27 months to 108 months, with the mean of 74 months. They were ineffectively treated with drugs or by desensitization therapy before, combined with bilateral inferior turbinate hypertrophy which were edema and pale and mucosa could not be shrank by ephedrine or $0.1 \%$ adrenaline, together with deflection of nasal septum. Postoperative follow-up lasted 16 to 30 months. With random sampling method, No. 6, 10, 12, 23, 25 and 31 of the experimental group were determined to be the numbers of ultrastructure TEM observation before and after operation, who were informed and signed informed consent.

\subsection{The Preparation of Electron Microscopy Specimens}

The preparation of electron microscopy specimens of inferior turbinate mucosa: before operation and one year after operation, a $1 \mathrm{~cm}$ incision was done in the front muscosa of right inferior turbinate, and a set of mucosa tissue about $0.3 \mathrm{~cm} \times 0.3 \mathrm{~cm} \times 0.2 \mathrm{~cm}$ was taken from it with small scissors for eyes and then rinsed with $0.9 \%$ normal saline, immediately fixed with glutaraldehyde-paraformaldehyde solution and $\mathrm{OsO}_{4}$ solution, progressively dehydrated with ethanol-acetone, embedded with epoxy resin 618 , and 
ultrathin section was dyed by uranium and lead, and observed with $\mathrm{Hu}-12 \mathrm{~A}$ electron microscope and Philips EM208 electron microscope.

\section{Results}

In this research, what could nasal mucosa of APR patients before operation be seen under electron microscope were: nasal mucosa ciliated columnar epithelium was nude, with few microvillus, no cilia; epithelial cells were shed; interstitial edema was obvious; there were plenty of eosinophil (EO), mast cells (MC) infiltration and a great deal of degranulation (extensive extracellular matrix particles, cytoplasmic vacuolization), and the uneven center crystal of grain density disappeared; there were lots of phagocyte, lymphocytes, plasma cells and a little inflammatory cell infiltration; collagen fiber disorderly ranged and ruptured; lamina propria gland was edema and glandular cells were edema and dysplasia; lumen was rich in secretory granules and inflammatory cells (as shown in Figures 1-3). The performance of the nasal mucosa one year after operation was: the nasal epithelial cells were still nude, and a few microvillus regenerated without cilia; interstitial edema was significantly improved; there was significantly reduced EO infiltration, of which the granule density was even and the center crystal was clear with steady structure; MC infiltration could still be seen; no granule was seen in extracellular matrix; there was no vacuolization in cytoplasmic; what could be seen were neutrophils, monocytes and a few phagocytes, lymphocytes and plasma cells; collagen fiber arranged orderly without any rupture; lamina propria gland edema disappeared, and glandular cells had normal structure, and plenty of orderly arranged microvillus were seen; lumen rarely secreted particles and inflammatory cells (see Figures 4-6).

Clinical curative effects: As for the major symptoms of APR patients, nasal congestion and runny nose are most greatly improved. Compared the preoperative nasal congestion and runny nose and the ones of the second year's postoperative follow-up, the differences were still significant. The curative effect of sneezing and nasal itching was unsatisfactory, and there was no difference when comparing the curative effects of the

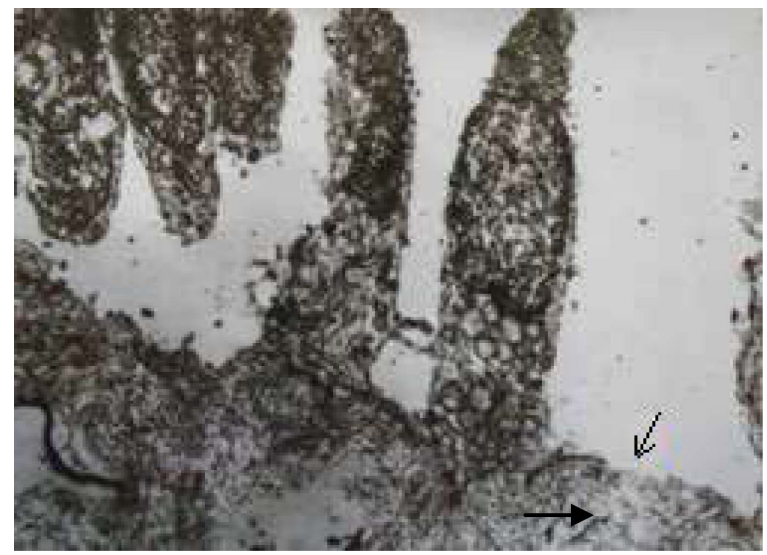

Figure 1. Preoperative nasal columnar epithelium without cilia, basement membrane of epithelial was edema and cytoplasm vacuolization cells was nude $\uparrow$, disordered arrangement of collagen fibers $\rightarrow \times 3000$. 


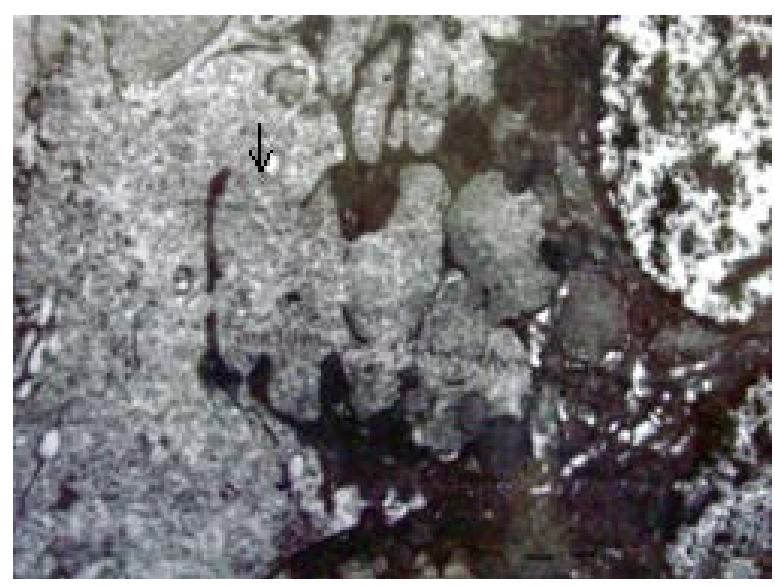

Figure 2. Preoperative glandular epithelial cell was edema and cytoplasm vacuolization and was like-“crater" change $\uparrow \times 4800$.

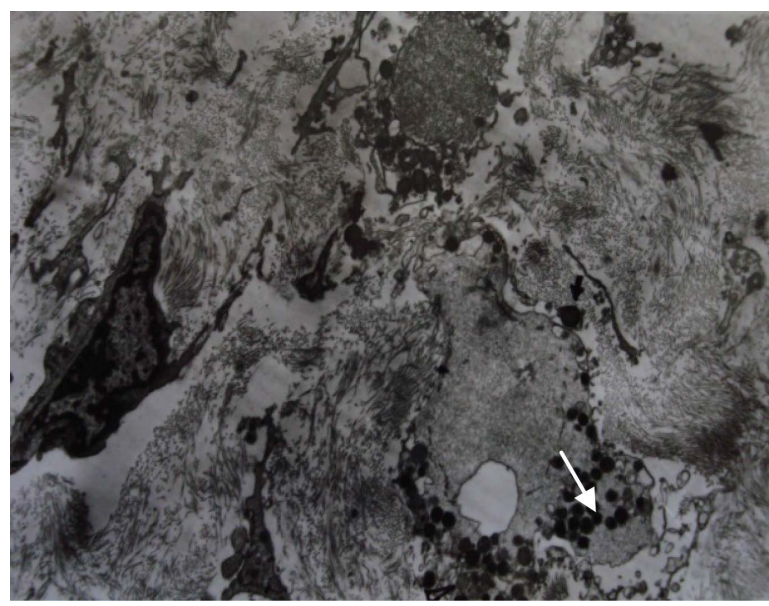

Figure 3. Preoperative the degranulation of EO and MC, granules crystalloid was lost. $\uparrow \times 3500$.

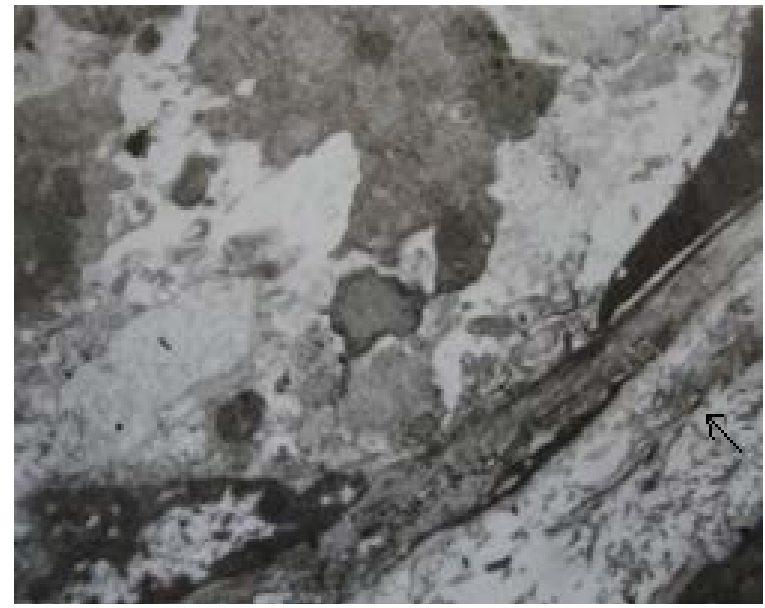

Figure 4. Postoperative nasal columnar epithelium still without cilia, basement membrane of epithelial cells still was nude, but collagen but collagen fibers turned into ordered. $\uparrow \times 7000$. 


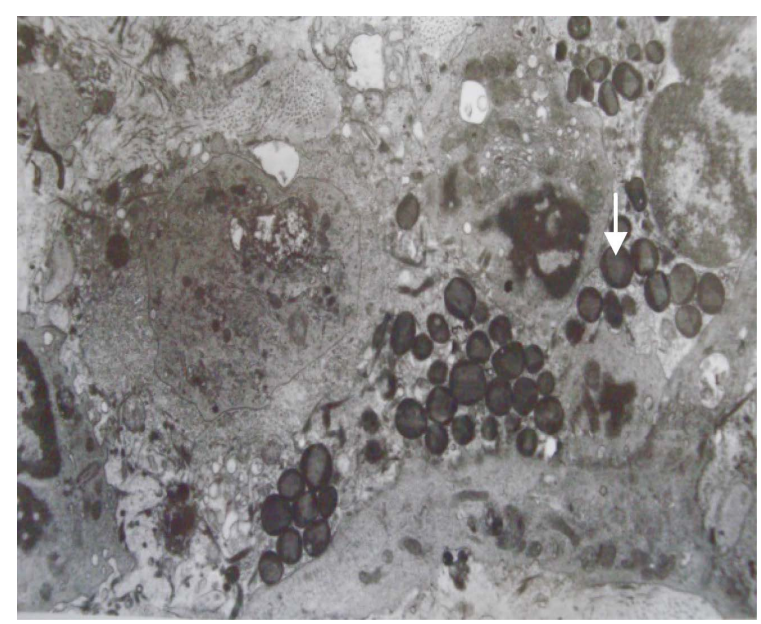

Figure 5. Postoperative the density of EO granules was consistent with its crystalloid $\uparrow \times 7000$.

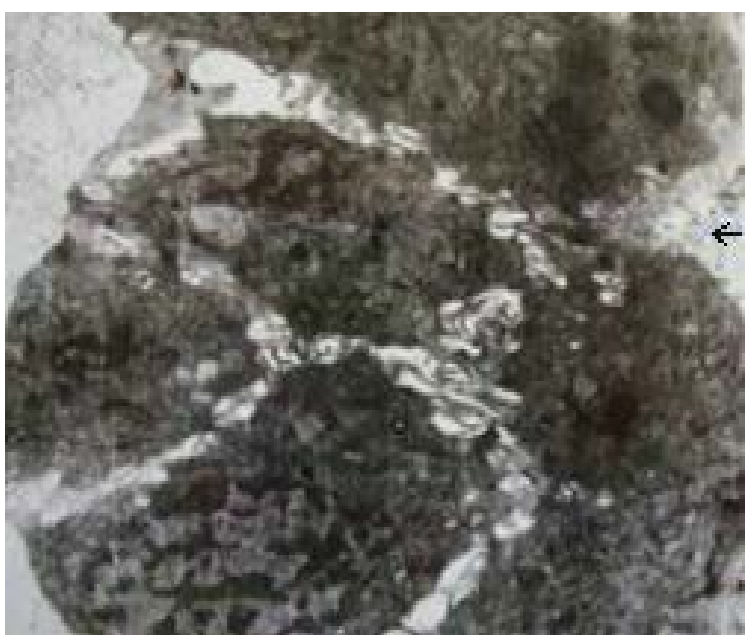

Figure 6. Postoperative construction of glandular epithelial cell was normal, and seldom granules was secreted, its superficies with plenty of microvillus $\uparrow \times 4800$.

second year's postoperative follow-up and the preoperative symptoms. The significant effective rate of 0.5 year after operation is $82.7 \%$, effective rate of $17.3 \%$, and total effective rate is $100 \%$; The significant effective rate of one year after operation is $46.7 \%$, effective rate of $53.3 \%$, and total effective rate is $100 \%$; The significant effective rate of two year after operation is $20.0 \%$, effective rate of $70.0 \%$, and ineffective rate is $10.0 \%$ [1].

\section{Discussion}

There are reports on the surgical treatment of APR from time to time, and the shortterm curative effect was satisfactory. For APR patients, operations cannot radically cure it, but improve patients' life quality. The diagnosis and therapy guideline and interpretation of allergic rhinitis [5]-[7] also refer that for the AR patients who are ineffectively 
treated by drugs or immunization therapy and whose major symptoms are nasal obstruction, radiofrequency operation of inferior nasal concha and inferior turbinate angioplasty plus emigration with mucosa reserved can be conducted. The non-immunological factors in AR pathogenesis have attracted much attention in recent decade, and these factors can cause and aggravate clinical symptoms, illuminating the mechanism of pathogenesis and aggravation when there is no allergen contact. This research chooses APR patients with deflection of nasal septumm and inferior turbinate hypertrophy to operate. By means of inferior turbinectomy and septoplasty to the subjects (36 cases of APR) [1], sensation-parasympathetic reflex was reduced, and so was glandular secretion, which forms scars of nasal mucosa and mucous membrane and related structural damage, causing the damage of nerve fiber endings widely distributed in mucous, the decrease of nasal mucosa's sensitivity, mucosal secretion and vascular permeability, and improving patients' nasal obstruction, and thus improving the clinical symptoms of APR.

There is infiltration of EO in both allergic rhinitis and non-allergic rhinitis, which is the basic pathological change of inflammatory response. However, eosinophil (EO) in $\mathrm{AR}$, a non-specific index in $\mathrm{AR}$, is still an index for diagnosing $\mathrm{AR}$, as $\mathrm{EO}$ infiltration and degranulation in AR patients' nasal mucosa are the features differing from other inflammatory response. Nevertheless, whether there is different ultrastructure of EO in $\mathrm{AR}$ and different $\mathrm{EO}$ in attacking period and stable period requires our further observation.

Many researches [8]-[11] have divided the histological features of APR patients' nasal mucosa into attacking period and remission period. 1) The ultrastructual features of nasal mucosa in attacking period are: Nasal mucosa indicates that epithelial cells are nude; partial epithelial cells are necrosis; cilia are lost, and microvillus are disorderly arranged; there is interstitial edema, swelling of mitochondria, significant infiltration of EO, MC, lymphocytes and plasma cells, increased membrane folds with pseudopod extending; there are a great deal of granules with varied density in EO and MC endochylema, with center crystal lost, significant vacuolization, and degranulation was common. Gland is edema, and gland cell dysplasia, with secretory granules in lumen. Collagen fibers fracture and disorderly arranged. 2) The ultrastructural features of nasal mucosa in remission period: Nasal muscosa displays that most epithelial cells are still nude, without any cilia, but orderly-arranged microvillus is seen in some parts. Interstitial edema is improved; there is $\mathrm{MC}, \mathrm{BO}$, neutrophil infiltration, reduced EO infiltration, complete $\mathrm{MC}$ and $\mathrm{EO}$ cell membrane, stable structure of cytoplasmic granules, clear center crystal. Swollen glands disappear; glandular epithelial cells have complete structure, and secretory granules reduce; there is monocyte and lymphocytic infiltration, while collagen fiber orderly arrange.

Researches indicate that the EO aggregation in respiratory mucosa does not cause airway hyperresponsiveness, but only activated EO aggregation can lead to related clinical symptoms [10] [11]. Characteristics of particles also indicate the activation degree of EO. In recent years, the observation of EO's ultrastructure has been the focus of na- 
tive and overseas scholars [9] [10]. In this research, in nasal mucosa, it can be that EO structure is stable, while stable MC structure, clear granule center crystal, without degranulation, and the reduced infiltration of lymphocyte are also the features of nasal mucosa's postoperative ultrastructure, which is the same as that of remission period. What distinguishingly differs is the great deal of degranulation of EO and MC, the disappearance or blurs of EO granule center crystal. It demonstrates that the nasal mucosa's histopathological features of APR patients after operation still stay in remission period during the follow-up, which is also consistent with the improvement of its clinical symptoms. EO granule center crystal also greatly varies along with the activation and stability of EO. When observing the nasal ultrastructure after operation in this research, we also observed that the stable structure of MC and lymphocytes is accompanied by the reduced EO infiltration and stable state of structure. It is manifested that the activation and stabilization of EO is significantly related to EO granule center structure's disappearance or clarity.

To treat APR with partial resection of the inferior turbinate and nasal septum operation has a reliable short-term curative effect. This research also achieved similar curative effects in treating APR with the two surgeries [1]. As for the four symptoms of APR, nasal obstruction and running nose were improved most greatly, while sneezing and rhinocnesmus were less improved. However, it is not the first choice in treating APR. This method is only applied to those who are ineffectively treated with drugs or by desensitization therapy, and combined with bilateral inferior turbinate hypertrophy, edema and pale and mucosa could not be shrank by ephedrine or $0.1 \%$ adrenaline, together with deflection of nasal septum. Their clinical symptoms have been short-term improved. The observation of the nasal mucosa's ultrastructure one year after operation indicates the same improvement of clinical symptoms in follow-up. As the postoperative time goes, nasal mucosa tends to "resume", and the ultrastructure of nasal mucosa displays to be that the nasal epithelial is still nude without the repair of cilia, but the degranulation disappears, and interstitial EO infiltration significantly reduces, neutrophil and mast cell infiltration becoming the major chronic inflammatory reactions. Besides, gland edema is also significantly improved and the structure resumes. For the treatment of APR with the methods presented by our research institution, in one year before and after the operation, ultrastructural changes of inferior turbinate mucosa tissues were observed from the preoperatively pathological changes of typical APR to the chronic inflammation with the primary infiltration of neutrophilic granulocyte and mast cells.

\section{References}

[1] Lin, W.N. and Yang, J.S. (2008) Allergic Perennial Rhinitis Treated by Inferior Turbinectomy and Septoplasty. Chinese Journal of Clinical Immunology and Allergy, 2, 37-40.

[2] Yang, J.S., Li, Z.C., Guo, Z.Y., et al. (1988) 1/3 Resection of Bilateral Inferior Turbinate Treating Allergic Rhinitis. Journal of Fujian Medical College, 4, 363-364.

[3] Shen, Z., Chen, W.W. and Deng, Y.X. (2001) The Clinical Observation of Allergic Rhinitis 
Symptoms Improved by Nasal Septum Operation. Clinical Otorhinolaryngology, 8, 370371.

[4] Zhou, B, Chen, X.Y., He, S.H., et al. (1998) Sphenopalatine and Anterior Ethmoidal Nerve Electrocauterizing and Inferior Turbinectomy Treating Allergic Perennial rhinitis. Chinese Journal of Otorhinolaryngology, 5, 318.

[5] Chinese Medical Association of Otorhinolaryngology (2005) Allergic Rhinitis Diagnosis and Treatment Principles and Recommended Solutions (2004, Lanzhou). Chinese Journal of Otorhinolaryngology, 40, 166-167.

[6] Rhinology Group of Otorhinolaryngology Head and Neck Operation Editorial Board, Rhinology Group of Otorhinolaryngology Head and Neck Operation Branch of Chinese Medical Association (2009) The Diagnosis and Treatment of Allergic Rhinitis (2009, Wuyi Mountain). Otorhinolaryngology Head and Neck Operation, 44, 977-978.

[7] Rhinology group of Otorhinolaryngology Head and Neck Operation Editorial Board (2009) The Interpretation of The Diagnosis and Treatment of Allergic Rhinitis. Otorhinolaryngology Head and Neck Operation, 44, 972-976.

[8] Xia, Y. and Zhu, L.X. (Overview) (1996) The Research Progress of Eosinophils of Allergic Rhinitis. Oversea Medicine.Otorhinolaryngology Fascicule, 20, 72-75.

[9] Zhao, C.Q., et al. (1997) TEM Observation of Nasal Eosinophil Degranulation Allergic Rhinitis. 2, 103-105.

[10] Lin, J.T., Hiroshi, I., Suzuki, T., et al. (1994) Eosinophilic Airway Inflammation and Airway Hyperresponsiveness. Chinese Journal of Tuberculosis and Respiratory Diseases, 17, 213215.

[11] Hamann, K.J., et al. (1991) In: Farmer, S.G., Hay, D.W., Eds., The Airway Epithelium: Physiology, Pathophysiology, and Pharmacology, Marcel Dekker, New York, 255-286.

Submit or recommend next manuscript to SCIRP and we will provide best service for you:

Accepting pre-submission inquiries through Email, Facebook, LinkedIn, Twitter, etc. A wide selection of journals (inclusive of 9 subjects, more than 200 journals)

Providing 24-hour high-quality service

User-friendly online submission system

Fair and swift peer-review system

Efficient typesetting and proofreading procedure

Display of the result of downloads and visits, as well as the number of cited articles

Maximum dissemination of your research work

Submit your manuscript at: http://papersubmission.scirp.org/

Or contact ijohns@scirp.org 